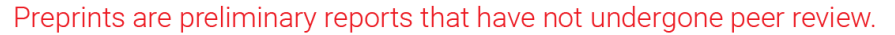 They should not be considered conclusive, used to inform clinical practice, or referenced by the media as validated information. \\ Estimation technique of global fringe visibility using in interferometers with adjustable visibility
}

fu-zhong bai

Inner Mongolia University of Technology https://orcid.org/0000-0003-0886-4551

Jiayi Chen

Inner Mongolia University of Technology

Xiaojuan Gao ( $\nabla$ joan789@163.com )

Inner Mongolia University of Technology

\section{Yongxiang $\mathrm{Xu}$}

Inner Mongolia University of Technology

\section{Research}

Keywords: Interferometer, Fringe pattern, Visibility estimation, Fourier-polar transform

Posted Date: August 20th, 2020

DOl: https://doi.org/10.21203/rs.3.rs-58924/v1

License: @ (i) This work is licensed under a Creative Commons Attribution 4.0 International License.

Read Full License 


\title{
Estimation technique of global fringe visibility using in interferometers with adjustable visibility
}

\author{
Fuzhong $\mathrm{Bai}^{1}$, Jiayi Chen ${ }^{1}$, Xiaojuan $\mathrm{Gao}^{2 *}$, Yongxiang $\mathrm{Xu}^{1}$ \\ *Correspondence:joan789@163.com (XJ Gao)
}

\begin{abstract}
In the accuracy measurement of phase from interferometers with adjustable fringe visibility, it needs to estimate the visibility of experimental patterns so as to obtain the interference patterns with the maximum visibility. We develop the Fourier-polar transform and combine the directional projection to estimate the global visibility of carrier fringe pattern. The technique is especially used for low-quality fringe pattern such as low contrast and low signal to noise ratio (SNR) that often appear in the interferometric experiment. An illustrative experiment based on the radial shearing interferometer is given. Results generated from this technique are compared with the derived values from theoretical model, and exemplary agreement between both is demonstrated.
\end{abstract}

Keywords: Interferometer; Fringe pattern; Visibility estimation; Fourier-polar transform

\section{Introduction}

Optical techniques such as digital phase-measuring interferometer [1], electronic speckle pattern interferometer [2] and fringe projection [3] are quite popular for non-contact measurements in the areas of experimental mechanics, profilometry and non-destructive testing and evaluation, and have been applied for measuring various physical quantities like vibration and displacement, strain, surface profile, depth, refractive index, optical metrology operation etc [4,5]. In all these techniques, the information about the measured physical quantity is stored in the phase or its associated derivatives of a fringe pattern. With image processing or signal analysis techniques the phase (and/or its derivatives) can be extracted from the fringe pattern.

Furthermore, compared with other optical techniques, the interferometer holds higher precision of measurement and thus is frequently applied in precise measurement and optical testing [6,7]. The fringe patterns are important output information of interferometers. Therefore, in order to realize higher measuring accuracy, fringe patterns with high quality need to be acquired. The influencing factors to fringe quality mainly involve noise and nonlinear response from the detector, intensity fluctuation from aperture diffraction, spatial resolution and gray level of image and fringe visibility as well. As is well known, the fringe visibility is one of the most important factors heavily affecting the measuring accuracy of interferometers [8,9]. Therefore, researchers go to great lengths to design an interferometer with ideal fringe visibility. In our previous works, two self-referencing interferometers respectively based on point diffraction and radial shearing structures have been built $[10,11]$, in which the circular path structure is used to realize common-path interference and the wavefront phase is extracted with phase-shifting technique or
Fourier transform method. The most attractive advantage of these interferometers is that the fringe visibility is adjustable and it is especially important to high accuracy measurement of interferometers. In addition to the above-mentioned devices, researchers have built a variety of interferometers with adjustable visibility [12-14]. Based on the special optical structure or incident wavefront types, researchers adjust the fringe visibility to satisfy the requirement of measurement. Through analyzing a series of recorded patterns during the measurement, the high visibility patterns can be generated. Furthermore, the low-quality fringe patterns frequently appear in the experimental tests of interferometers.

According to the definition of fringe visibility, the fringe visibility can be calculated through finding the maximum and minimum intensity values in the pattern [15]. However, due to the effect of noise from the detector, fluctuation of intensity distribution and other environmental disturbs, the practicability of this technique is limited or only can be used in estimating local visibility. In present, the analysis of fringe pattern focuses on fringe filtering [16], orientation estimation [17], fringe visibility enhancement [18], fringe pitch detection [19], fringe shift analysis [20,21] and so on.

In view of our analysis, the estimation of global fringe visibility available for low-quality pattern will be urgently needed to be studied. We develop a new technique of Fourierpolar transform and use it to estimate fringe visibility of lowquality pattern. The principle of technique is introduced with the aid of simulated fringe patterns analysis. We demonstrated this technique on analyzing experimental fringe patterns generated from a radial shearing interferometer with adjustable fringe visibility.

\section{Methods}




\section{Fringe visibility in two-beam interferometer}

The mathematical form of a typical fringe pattern from twobeam interference is given by

$$
I=I_{1}+I_{2}+2 \sqrt{I_{1} I_{2}} \cos \varphi
$$

where $I_{1}$ and $I_{2}$ indicate the intensity distribution of the respective waves, and $\varphi$ is the phase difference between two interfering waves. All of them are the function of $x$ and $y$ that refer to the spatial coordinates or pixels along the horizontal and vertical directions.

In linear optical interferometers like the Michelson interferometer, interference manifests itself as intensity oscillations over space, also called fringes. Under these circumstances, the interferometric visibility is also known as fringe visibility. For this type of interference, in terms of the amplitude envelope of the fringe intensity and the average intensity over a given space domain, the visibility is written as $[22,23]$

$$
V=\left(I_{\max }-I_{\min }\right) /\left(I_{\max }+I_{\min }\right)
$$

where $I_{\max }$ and $I_{\min }$ are the maximum and minimum intensities respectively. For illustration, fringe patterns and their amplitude envelope are shown in Fig.1. If the two waves consist of only single wavelength of the same polarization, then the predicted visibility will be

$$
V=2 \sqrt{I_{1} I_{2}} /\left(I_{1}+I_{2}\right)
$$

Fringe visibility is scaled from 0 to 1 , where 0 means no fringes and 1 means perfect visibility [5]. According to the above definition of visibility, Eq.(1) is rewritten as

$$
I=I_{0}+I_{0} V \cos \varphi
$$

where $I_{0}=I_{1}+I_{2}$

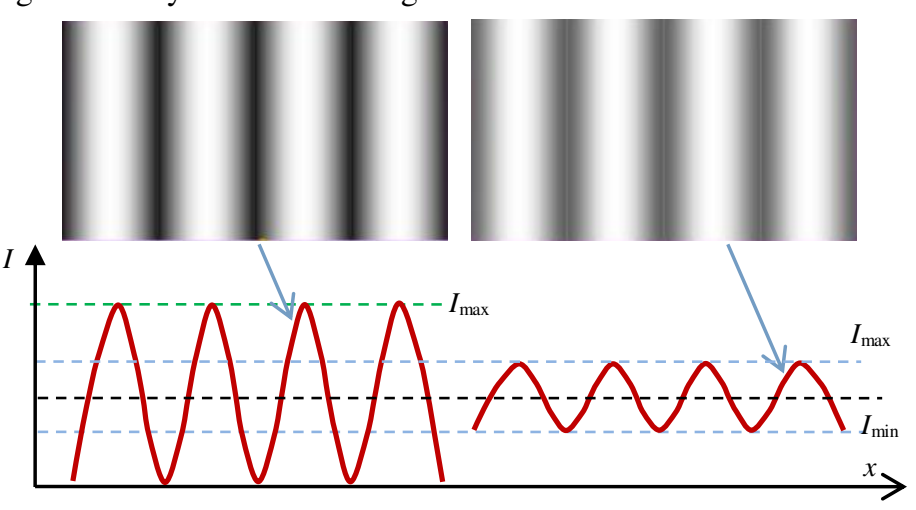

Fig. 1. Fringe patterns and their amplitude envelopes.

From Eq. (2), the fringe visibility can be estimated in terms of the observed intensity maxima and minima in an interference pattern. In fact, the intensity of optical beam is often nonuniform distribution, and the noise from the detector and unstable laser intensity will also affect the intensity from each pixel in the interference pattern deviate from the ideal intensity. Therefore, Eq. (2) cannot be directly applied to the experimental patterns to calculate the fringe visibility.

\section{Fringe direction determination based on Fourier-polar transform}

By introducing tilt modulation into two interfering beams, a carrier fringe pattern can be generated. In the case of no phase object introduced, according to Eq.(4) the carrier fringe pattern is written as

$$
I=I_{0}+I_{0} V \cos (2 \pi f x \cos \theta+2 \pi f y \sin \theta)
$$

where $f$ is the carrier frequency, and $\theta$ is the angle between the fringe direction and the $y$ axis of image. Assumed that $\theta=70^{\circ}$ and $V=0.8$, a frame of carrier fringe pattern with circular aperture and spatial resolution of $128 \times 128$ pixels is simulated and shown in Fig. 2(a).

The power spectrum of the carrier fringe pattern is expressed as $P(u, v)=|F(u, v)|^{2}$, where $F(u, v)$ is the Fourier transform of Eq.(5), and $u$ and $v$ represent the coordinates in the frequency domain. From the power spectrum as shown in Fig. 2(b) it can be concluded that the energy spread direction in the power spectrum is in accordance with the normal direction of fringe pattern. Therefore, the global fringe direction can be calculated from the power spectrum.

For ease of analysis, the power spectrum is transformed from Cartesian coordinate ( $u-v$ system) to polar coordinate $(r-\theta$ system). The conversions relationship is given by

$$
r=\sqrt{u^{2}+v^{2}}, \quad \theta=\arctan (v / u)
$$

where the meaning of $r$ and $\theta$ are described in Fig.3(a). The power spectrum under polar coordinate is expressed as $P(r, \theta)$. 


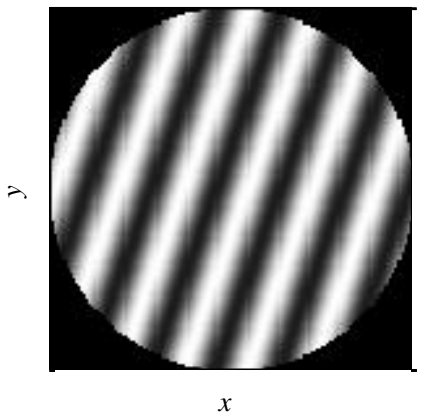

(a)

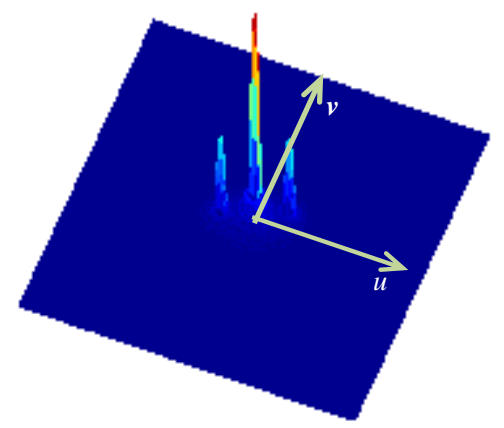

(b)

Fig. 2. Simulated fringe pattern (a) and its power spectrum (b).
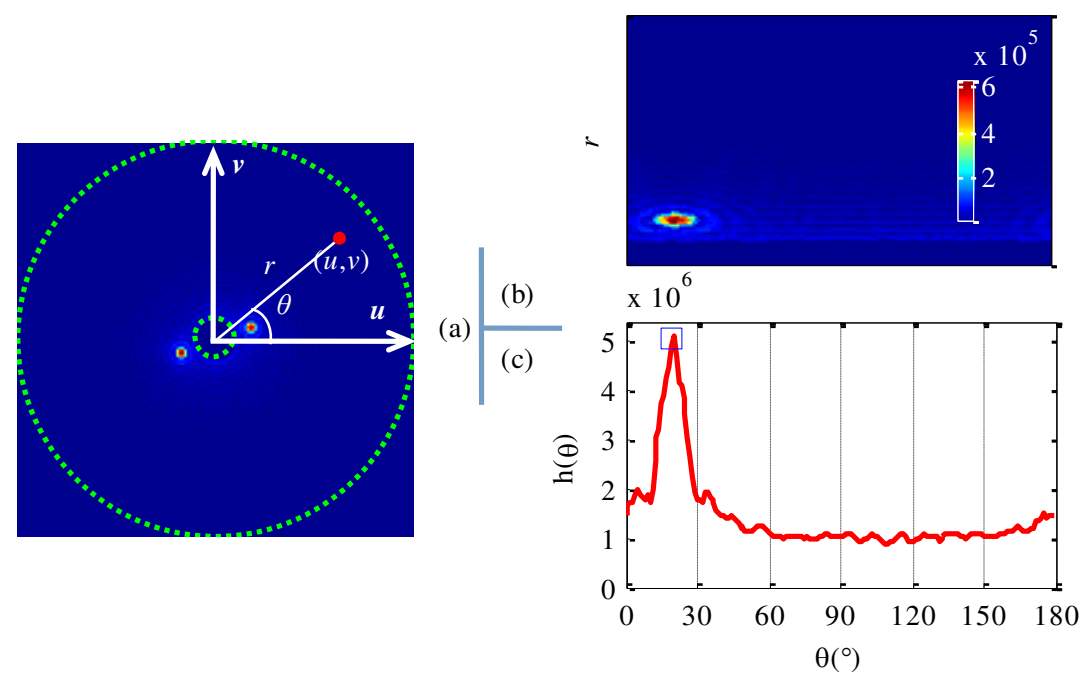

Fig. 3. Illumination of Fourier-polar transform. (a) and (b) power spectrums in Cartesian and polar coordinates, (c)total energy as a function of the polar angle.

Due to the energy at the zero frequency being generally larger, the data within the zone defined by $r \in[\varepsilon \rho, \rho]$ in power spectrum are implemented in the processing of polar coordinate transform, where $\rho$ is the half size of the power spectrum and $\varepsilon$ is set to 0.1 . The annular zone within dotted circles is plotted in Fig. 3(a). With the change of $\theta$ we can calculate the total energy along different direction in polar coordinates by using the following equation,

$$
h(\theta)=\sum_{r=0.1 \rho}^{\rho} P(r, \theta) .
$$

Therefore, we obtain the Fourier-polar transform result of the fringe pattern. Because the power spectrum is symmetry around the zero frequency, only half of $P(u, v)$, i.e., $\theta \in\left[0^{\circ}, 180^{\circ}\right)$, is needed to implement the polar coordinate transform.

Fig. 3(b) is the power spectrum under polar coordinate, namely, the polar coordinate transform of Fig.3(a). The accumulation along the $r$ axis in Fig. 3(b) can obtain $h(\theta)$ that is shown in Fig. 3(c). The $\theta_{\mathrm{m}}$ corresponding to the peak value in $h(\theta)$ expresses the normal direction of fringes, and so the fringe direction is $\theta_{0}=90^{\circ}-\theta_{\mathrm{m}}$. For the simulated pattern shown in Fig. 2(a), the calculated direction equals $69.41^{\circ}$ and the error equals $-0.59^{\circ}$.

\section{Fringe visibility estimation based on directional projection}

According to the calculated fringe direction, the directional projection of original pattern is implemented with the following equation $[24,25]$,

$$
R\left(x^{\prime}\right)=\int I\left(x^{\prime} \cos \theta_{0}-y^{\prime} \sin \theta_{0}, x^{\prime} \sin \theta_{0}+y^{\prime} \cos \theta_{0}\right) d y^{\prime}
$$

Two coordinate systems are related by a rotation transform that is given by

$$
\left[\begin{array}{l}
x^{\prime} \\
y^{\prime}
\end{array}\right]=\left[\begin{array}{cc}
\cos \theta_{0} & \sin \theta_{0} \\
-\sin \theta_{0} & \cos \theta_{0}
\end{array}\right]\left[\begin{array}{l}
x \\
y
\end{array}\right]
$$


The directional projection of the fringe pattern shown in Fig. 2(a) is shown in Fig. 4(a).

The number of valid pixels (i.e., $N\left(x^{\prime}\right)$ ) within the aperture projected on the $x$ ' axis is plotted in Fig. 4(b). It shows that the pixel's numbers in different colummns along the fringe direction are not equal. Therefore, in order to perfectly represent the distribution of fringe intensities, the mean projection intensities are further calculated with $R\left(x^{\prime}\right) / N\left(x^{\prime}\right)$ and are shown in Fig. 4(c). With the least square algorithm the mean intensities are fitted to an cosine function, so we can obtain the fringe visibility. For this simulated pattern, the estimated visibility equals 0.79 , which closely agrees with the given value $(V=0.8)$.
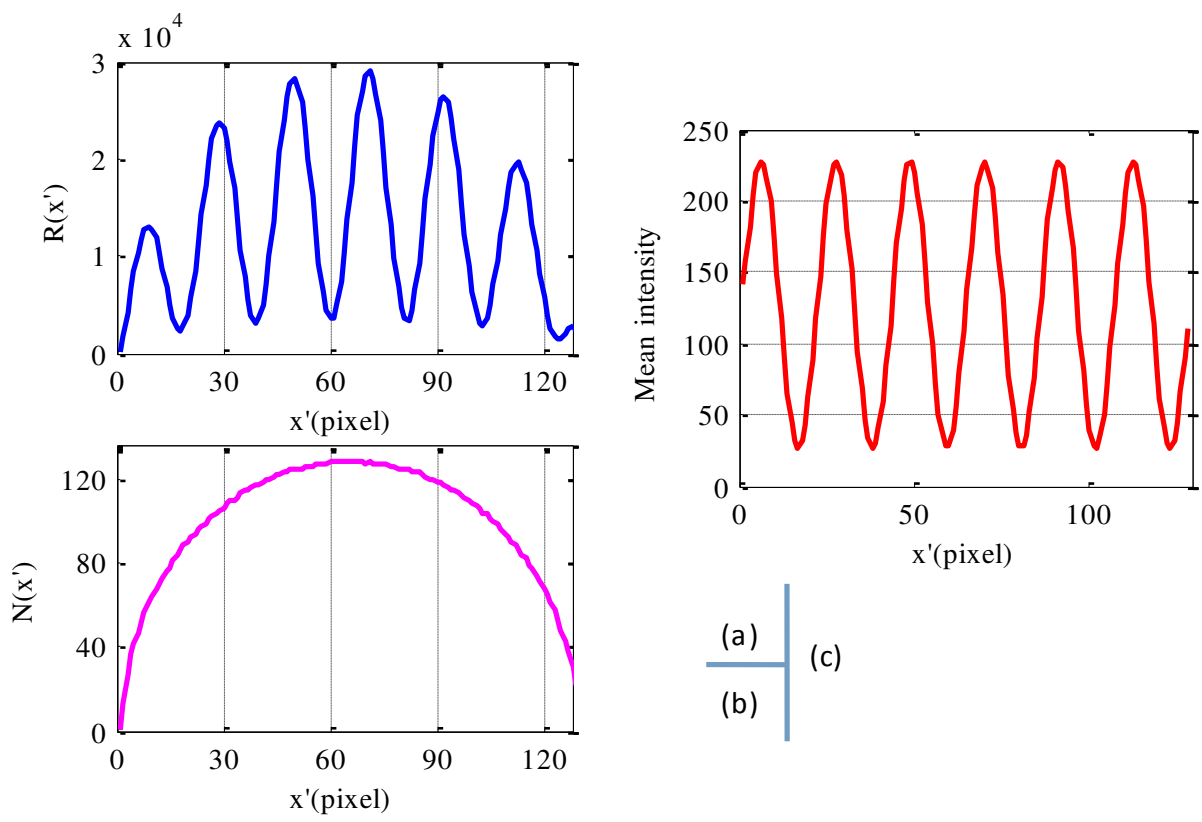

Fig. 4. Accumulated intensities (a), pixel's number (b) and mean intensities (c) along the fringe direction.

\section{Experiments}

\section{Optical setup of radial-shearing interferometer with adjustable visibility}

A common-path radial shearing interferometer with adjustable fringe visibility is design and shown in Fig. 5(left). The optical structure includes a polarizing beam splitter (PBS), two reflective mirrors (M1 and M2), and two lenses (L1 and L2). The focal lengths of two lenses composed of telescope system are $f_{1}=250 \mathrm{~mm}$ and $f_{2}=300 \mathrm{~mm}$, respectively. The collimated and expanded linearly polarized light from the laser passes through the HWP and then introduces into the radial-shearing system. The incident beam is split into two linearly polarized beams with orthogonal polarization. The reflected beam traverses the path PBS-L1-M1-M2-L2-PBS, and its aperture is magnified because $f_{1}$ is less than $f_{2}$. Similarly, the transmitted beam traverses along the opposite path, and the aperture is demagnified. The magnified and the demagnified beams are entirely reflected and transmitted through the PBS, respectively. Through the QWP that its fast axis orientates at $45^{\circ}$ with respect to two linearly polarized beams, two beams are converted into circularly polarized lights, and then generate interference after passing through the polarizer.
The laser with wavelength of $632.8 \mathrm{~nm}$ and an 8-bit CCD camera are used in the setup. By appropriately tilting the mirrors (M1 or M2) the pattern involving a few of fringes can be generated. By rotating the HWP from $5^{\circ}$ to $85^{\circ}$ with a step of $5^{\circ}$, a total of seventeen frames of fringe patterns with the spatial resolution of $128 \times 128$ pixels are recorded, and six frames of them are shown in Fig. 5(right). When $\alpha=0^{\circ}$ or $90^{\circ}$, no interference fringes are generated.

\section{Mathematical model of fringe visibility from the experimental interferometer}

For simplicity, assumed that the intensities of all beams are uniform and the intensity of the incident light before the PBS is expressed as $I_{0}$. The angle of the HWP with respect to horizontal direction is $\alpha$. The intensities of transmitted and reflected beams through the PBS are respectively expressed as

$$
I_{\mathrm{t}}=I_{0} \cos ^{2} \alpha, \quad I_{\mathrm{r}}=I_{0} \cos ^{2}(\pi / 2-\alpha)=I_{0} \sin ^{2} \alpha
$$

Exiting from the radial shearing system, the transmitted and reflected beams are respectively demagnified and magnified. The intensities of two beams within their common areas imaged onto the camera are defined respectively as $I_{\mathrm{t}}^{\prime}$ and $I_{\mathrm{r}}^{\prime}$.

$$
I_{\mathrm{t}}^{\prime}=s^{2} I_{0} \cos ^{2} \alpha, \quad I_{\mathrm{r}}^{\prime}=I_{0} \sin ^{2} \alpha / s^{2}
$$




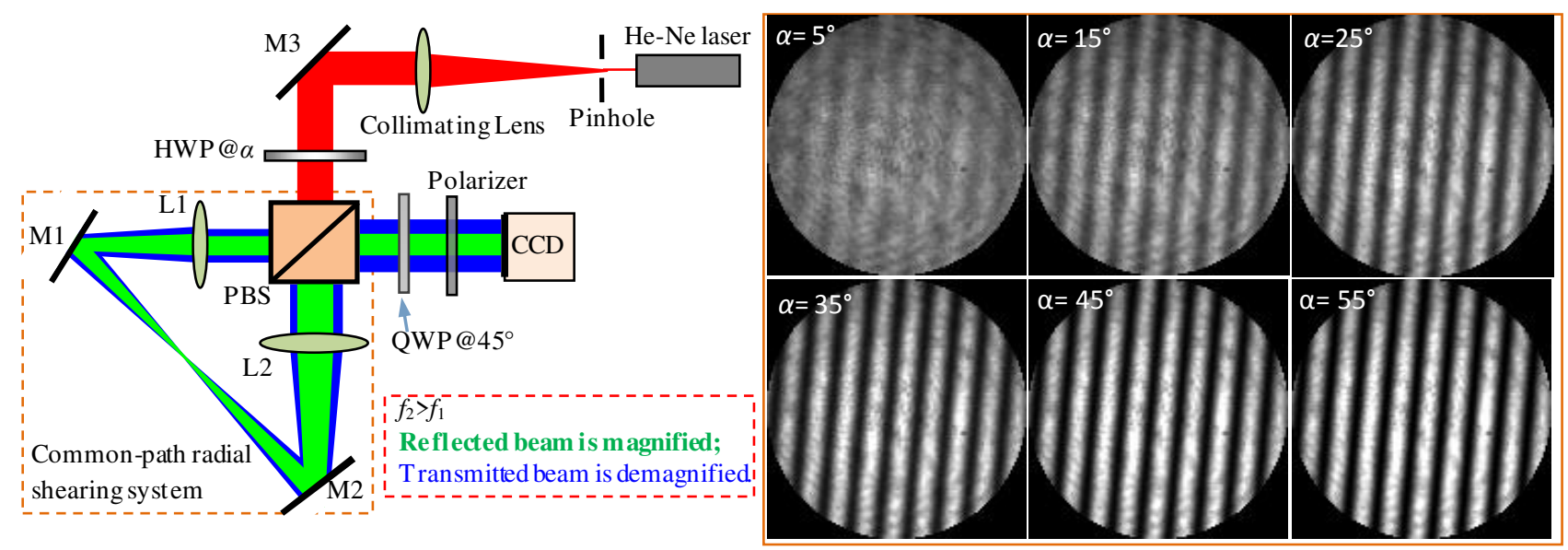

Fig. 5. Experimental set up of common-path radial shearing interferometer and the experimental patterns under different rotating angle of HWP. HWP, half wave plate; PBS, polarizing beam splitter; L1-L2, lenses; M1-M3, mirrors; QWP, quarter-wave plates

where $s=f_{2} / f_{1}>1$ that represents the shearing ratio of the setup. According to Eq. (3) and Eq. (11) the relation between $V$ and $\alpha$ is given by

$$
V=\frac{2 \cos \alpha \sin \alpha}{s^{2} \cos ^{2} \alpha+\sin ^{2} \alpha / s^{2}}=\frac{2}{s^{2} / \tan \alpha+\tan \alpha / s^{2}}
$$

From Eq. (12) we can obtain the maximal visibility (i.e., one) when $\alpha$ and $s$ satisfies the following relation,

$$
s^{2} \cos ^{2} \alpha=\sin ^{2} \alpha / s^{2}
$$

Eq. (13) can be derived on the basis of $I_{\mathrm{t}}^{\prime}=I_{\mathrm{r}}^{\prime}$. Furthermore, we can obtain

$$
\alpha=\arctan \left(s^{2}\right)
$$

\section{Visibility estimation of experimental fringe patterns}

The fringe directions of all experimental patterns can be determined through finding the peak position in $h(\theta)$ as shown in Fig.6. In this experiment, all recorded patterns hold the same fringe direction. It can also be seen from the calculated results as shown in Fig.7. For the low-quality patterns (such as $\alpha \leq 15^{\circ}$ or $\alpha \geq 85^{\circ}$ ), the calculated values only deviate 0.224 degree (namely 0.0039 radian) with the values calculated for the higher quality patterns. This deviation value is equal to the designed step of $\theta$ in the computer programming. In addition, the greatly consistent results shown in Fig.7 indicate that the proposed technique holds great superiority to analyze the lowquality fringe patterns. Along the calculated direction, the mean projection intensities of all experimental patterns are calculated. From the waveform curves as shown in Fig. 8 we can see that these approximate cosine signals fluctuate around a constant gray value (about 110), and the modulation intensities gradually increases while $\alpha$ varies from $5^{\circ}$ to $55^{\circ}$.
Furthermore, with the least square algorithm the projection curves are respectively fitted to cosine functions, the respective visibilities are estimated, which are shown in Fig. 9 with some square labels. For this optical setup with $s=1.2$, according to Eq. (12) one can derive the relation of $V$ and $\alpha$, which is also plotted in Fig.9 with solid line. The changing trends of estimated and derived visibilities are in good agreement, and the maximal visibility appears at $\alpha=55^{\circ}$ for both cases.

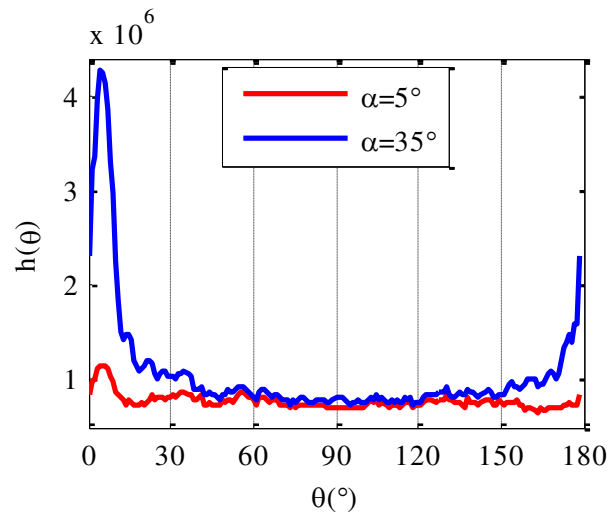

Fig.6. $\mathrm{h}(\theta)$ curves of two patterns shown in Fig.5(right).

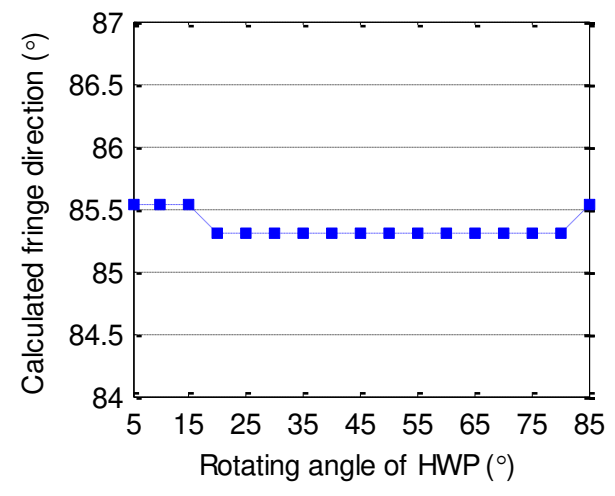

Fig. 7. Fringe directions calculated for all experimental patterns. 


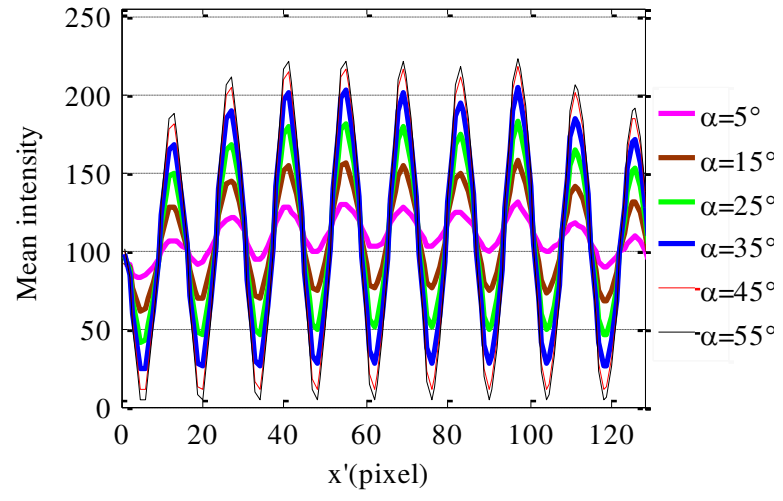

Fig. 8. Mean projection intensities of six patterns shown in Fig.5(right)

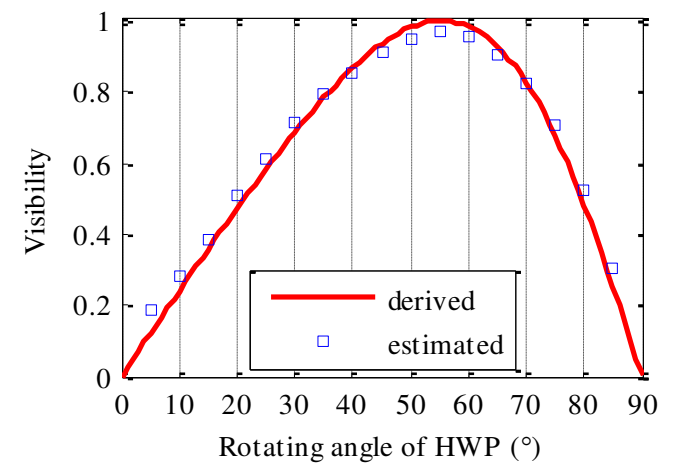

Fig. 9. The est imated visibility from experimental patterns and the derived visibility from theoretical model.

\section{Conclusions}

We have demonstrated a technique of Fourier-polar transform to estimate the global visibility of low-quality interference fringe pattern. With the home-built common-path radialshearing interferometer the proposed technique is tested experimentally. The fringe visibility estimated from this technique is compared with derived visibility values, and exemplary agreement between both is demonstrated. Without any requirement of image preprocessing such as filtering and thresholding operation, the proposed technique can determine the fringe direction from the power spectrum under polar coordinate system. Furthermore, due to the use of whole image information to determine fringe direction and the directional projection along the fringe direction, the new technique can reduce the effect of random noise, non-uniform intensity distribution and laser fluctuation to the greatest extent. Therefore, the proposed technique can be used to lowquality fringe pattern with good accuracy and reliability.

\section{Abbreviations}

SNR: signal to noise ratio

\section{Acknowledgements}

Not applicable.

\section{Authors' contributions}

FZ Bai conceived the presented idea. XJ Ga o developed the methodology and wrote the paper. JY Chen and YX Xu collected and a nalyzed the data. FZ Bais upervised the entire work. All authors read and approved the final manuscript.

\section{Funding}

The research is funded by the National Natural Science Foundation of China under Grant 51765054, Na tural Science Foundation of Inner Mongolia of China (2020LH06002) and the Science Foundation of Inner Mongolia University of Technology of China under Grant X201703.

\section{Availability of data and materials \\ Not a pplicable.}

\section{Competing interests}

The a uthors declare that they have no competing interests.

\section{Author details}

${ }^{1}$ College of Mechanical Engineering, Inner Mongolia University of Technology, Huhhot 010051, China. ${ }^{2}$ College of Astronautics, Inner Mongolia University of Technology, Huhhot 010051, China.

\section{References}

1. Y. Ishii, J. Chen, K. Murata, Digital phase-measuring interferometry with a tunable laser diode, Opt. Lett. 12(4)(1987)233-235.

2. P.K. Rastogi, Digit al speckle pattern interferometry and related techniques, Chichester: JohnWiley; 2000.

3. J.L. Lai, J.X. Li, C.Q. He, F Liu, A robust and effective phase-shift fringe projection profilometry method for the extreme intensity, Optik, 179 (2019) 810-818.

4. Dalip Singh Mehta, Mohammad Inam, Jai Prakash, A. M. Biradar, Liquid-crystal phase-shifting lateral shearing interferometer with improved fringe contrast for 3D surface profilometry, Appl. Opt. 52(25)(2013) 6119-6125.

5. G.Rajshekhar, P.Rastogi, Fringe analysis: Premise and perspectives, Opt. Laser Eng. 50(2012) iii-X.

6. J.X Peng, Z. Chen, Q.Z. Yuan, X.L. Feng, Precision measurement of electrical charge in a hybrid optomechanical system with Michelson interferometer, Opt. Commun. 458 (2020).

7. Y.J. Zhu, J.X. Na, W.Q. Pan, A.H. Li, S.H. Yin, Universal interferometer based on dynamically-adjusted fringe periods, Opt. Precision Eng. 20(1) (2012) 109-117.

8. Y.Kim, K. Hibino, N. Sugita, M. Mit suishi, Design of phase shifting algorithms: fringe visibility maximum, Opt. Express 22(15) (2014)18203-18213.

9. A. Sicardi-Segade, A. Martínez-García, N.I. Toto-Arellano, J. A. Rayas, Analysis of the fringes visibility generated by a lateral cyclic shear interferometer in the retrieval of the three-dimensional surface information of an object, Optik 125(3) (2014) 1320-1324.

10. Y.X. Xu, Y. Wang, Z. Liu, X.Q. Wang, Circular-path polarization point- diffraction interferometer and analy sis of the fringe parameters, Optik (158)(2018)915-922.

11. N.T. Gu, L.H. Huang, Z.P. Yang, C.H. Rao, A single-shot commonpath phase-stepping radial shearing interferometer for wavefront measurements, Opt. Express 19(5)(2011)4703-4713.

12. S.Karepov, N.T. Shaked, T. Ellenbogen, Off-axis interferometer with adjust fringe visibility based on polarization encoding, Opt. Lett. 40(10) (2015) 2273-2276.

13. D.S. Mehta, M. Inam, J. Prakash, Liquid-crystal phase-shifting lateral shearing interferometer with improved fringe visibility for 3D surface profilometry, Appl.Opt. 52 (25) (2013) 6119-6125. 
14. D.D. Wang, Y.Y. Yang, C. Chen, Y.M. Zhuo. Point diffraction interferometer with adjust able fringe visibility for testing spherical surfaces, Appl. Opt. 50(2011)2342-2348.

15. C. Huang, Effects of light intensity on visibility of interference fringe, J Shant ou Univ-Natural Science 16(1) (2001)68-71.

16. J. Vargas, C. Sorzano, J.A. Quiroga, J.C. Estrada, J.M. Carazo, Fringe pattern denoising by image dimensionality reduction, Opt. Lasers Eng. 51(2013)921-928.

17. Q. Sun, S.J. Fu, Comparative analysis of gradient-field-based orientation estimation methods and regularized singular-value decomposition for fringe pattern processing, Appl. Opt. 56(27)(2017) 7708-7716

18. L.Z. Cai, Q. Liu, X.L.Yang. A simple method of visibility enhancement and extremum extraction for interference fringes, Opt. Laser T echnol. 35(4) (2003) 295-302.

19. Y.X. Wang, F.Z. Bai, X.J. Gao, Y. Wang, Comparison of Spacing Detection Algorithms for Optical Straight Fringes Images, Proc SPIE 11053(2019) 110532W(8pages).
20. M. Farooq, A. Aslam, B. Hussain, G. Hussain, I. Masroor, A Comparison of image processing techniques for optical interference fringe analysis, Photonic Sensors 5(4) (2015) 304-311.

21. F.Z. Bai, F. Han, Y.X. Xu, X.Y. Bao, S.M. Gan, Modified Fouriertransform method for phase-shift calibration, Opt. Lasers Eng. 49(7)( 2011)932-936.

22. A.Y. Xu, Light line degree of visibility of interference fringe. Technology, 4(11)(2011)112-114.

23. A.M. Yan, X.Z. Wu, H. Liu, Quantitative theory of X-ray interferometers based on dual phase grating: fringe period and visibility, Opt. Express 16 (18)(2018) 23141-23155.

24. L.H. Ren, K. Liu, H.Y. Zhang. Rectangle detection of gray projection integral extreme value method, ComputerEng. 38(8) (2012)159-163.

25. T.Y. Zhang, F.Z. Bai, X.Y. Song XY, J.X. Wu, S.M. Gan, Image measuring method for width of straight-edge object, Laser \& Infrared 44(2) (2014)221-225 
Figures

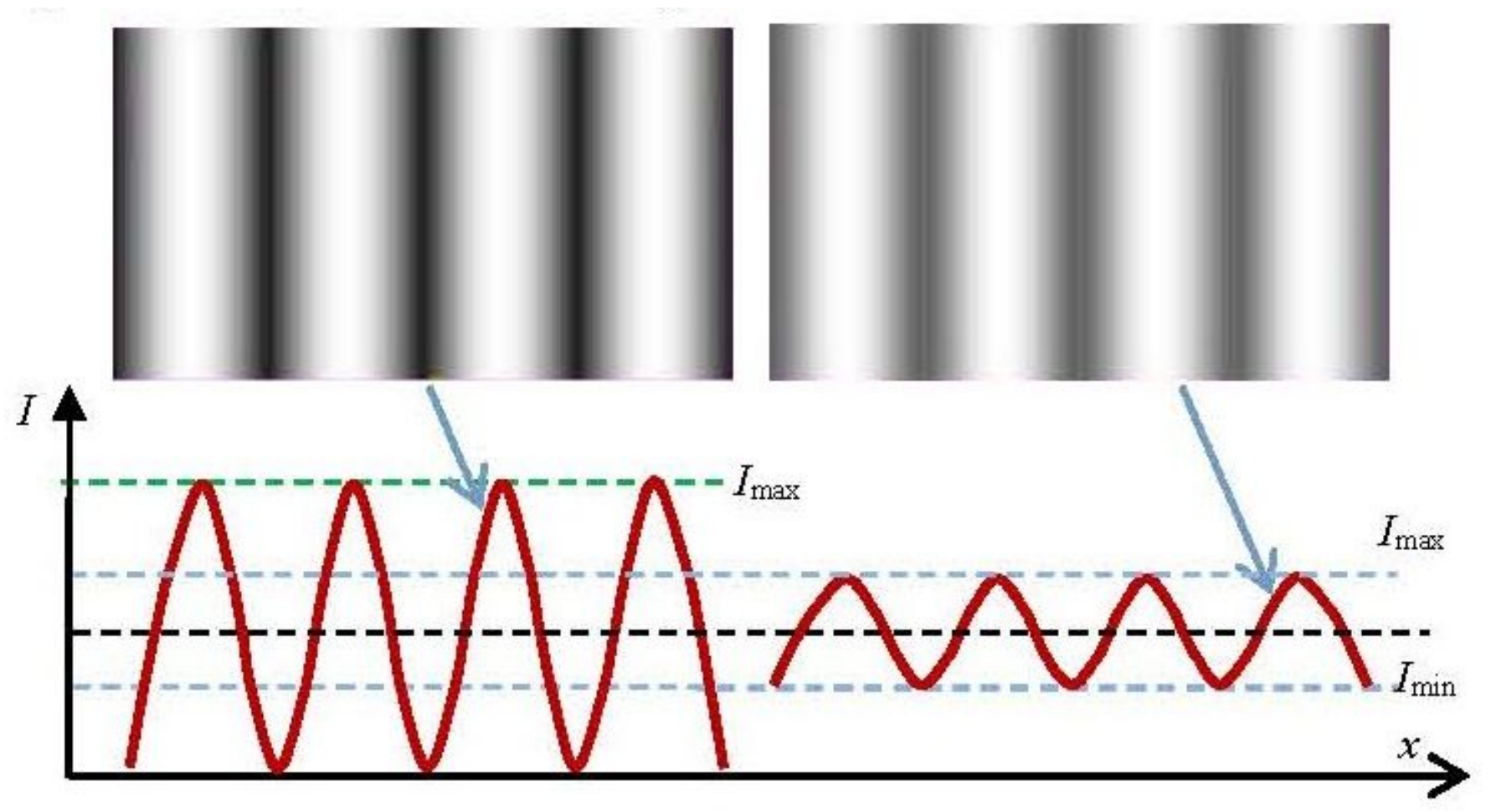

Figure 1

Fringe patterns and their amplitude envelopes.

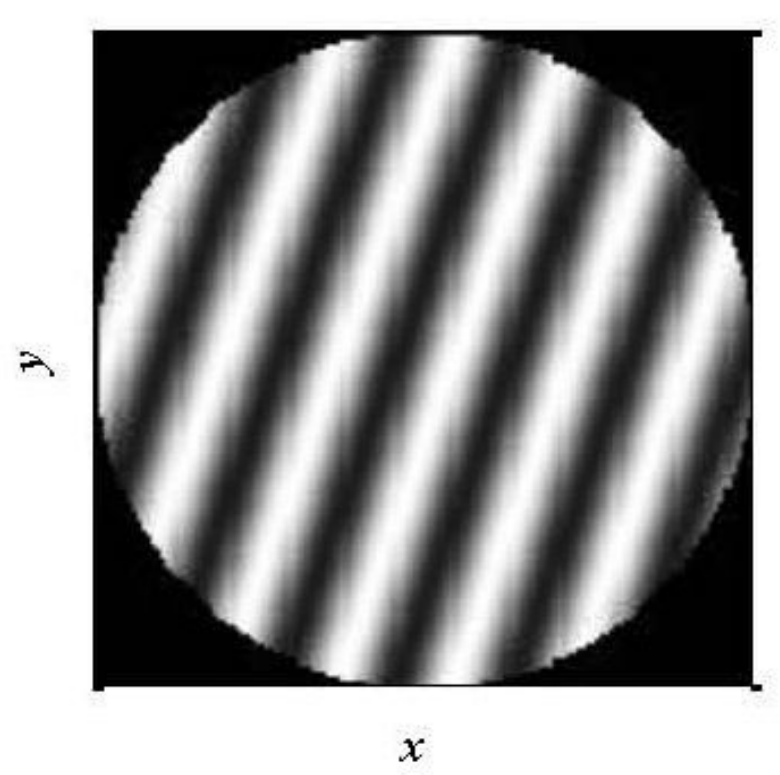

(a)

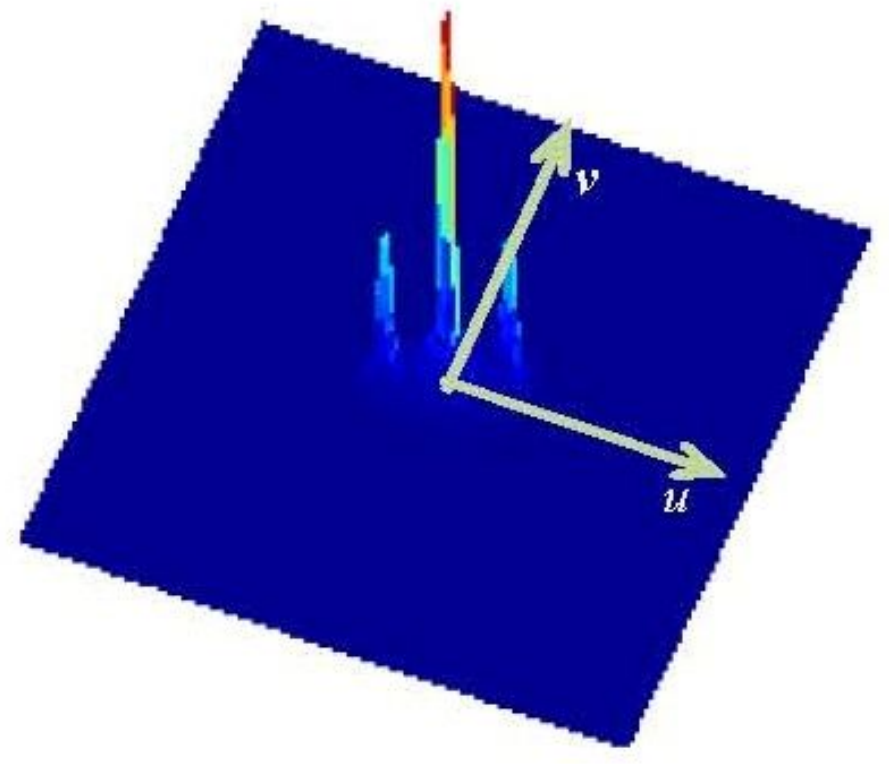

(b)

Figure 2

Simulated fringe pattern (a) and its power spectrum (b). 

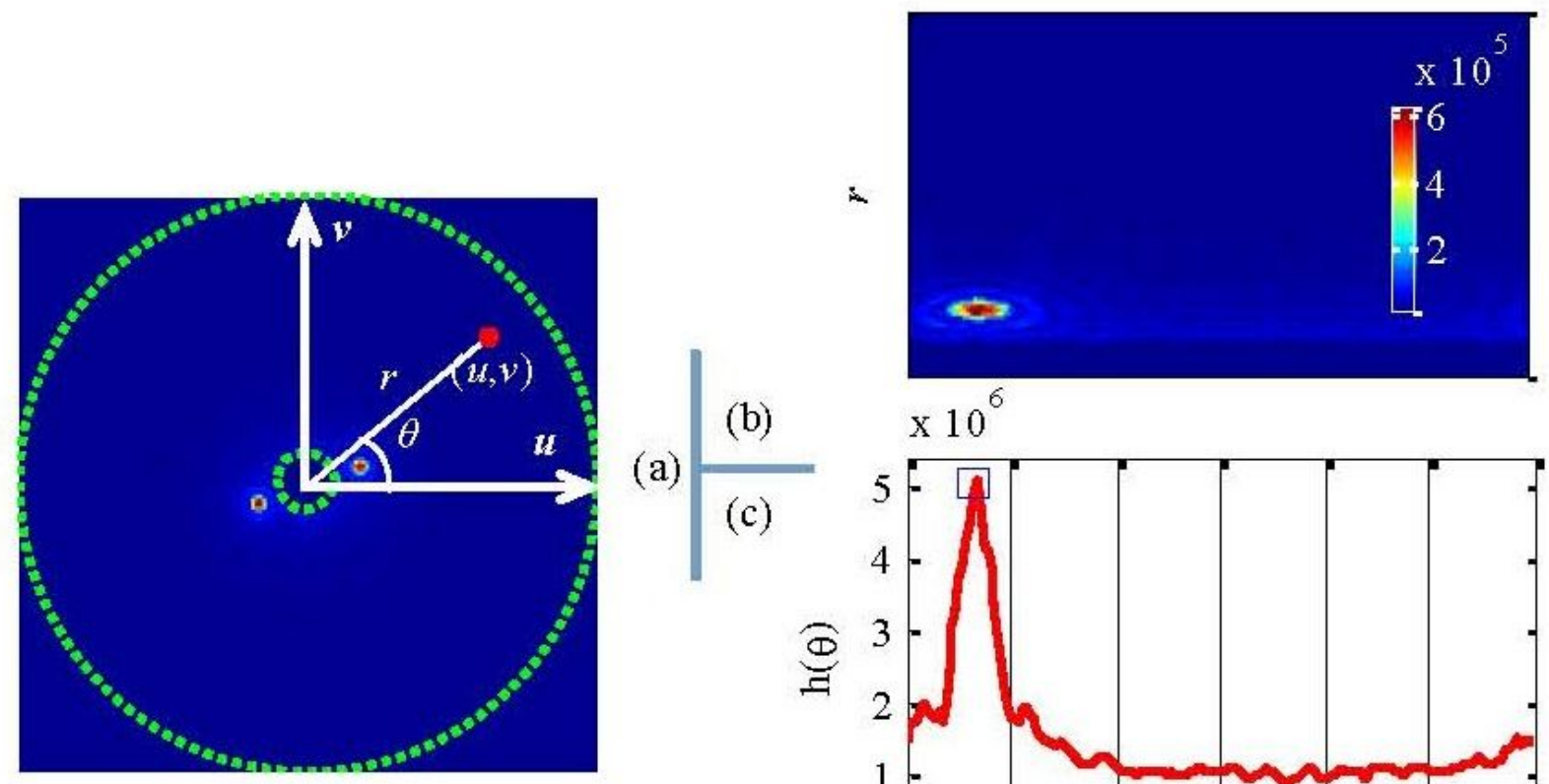

(a)

(b)

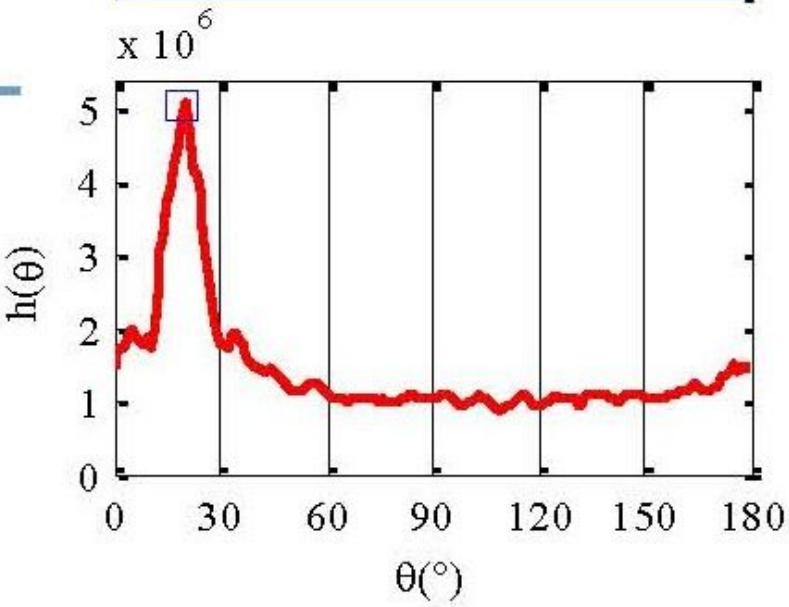

Figure 3

Illumination of Fourier-polar transform. (a) and (b) power spectrums in Cartesian and polar coordinates, (c) total energy as a funct ion of the polar angle. 

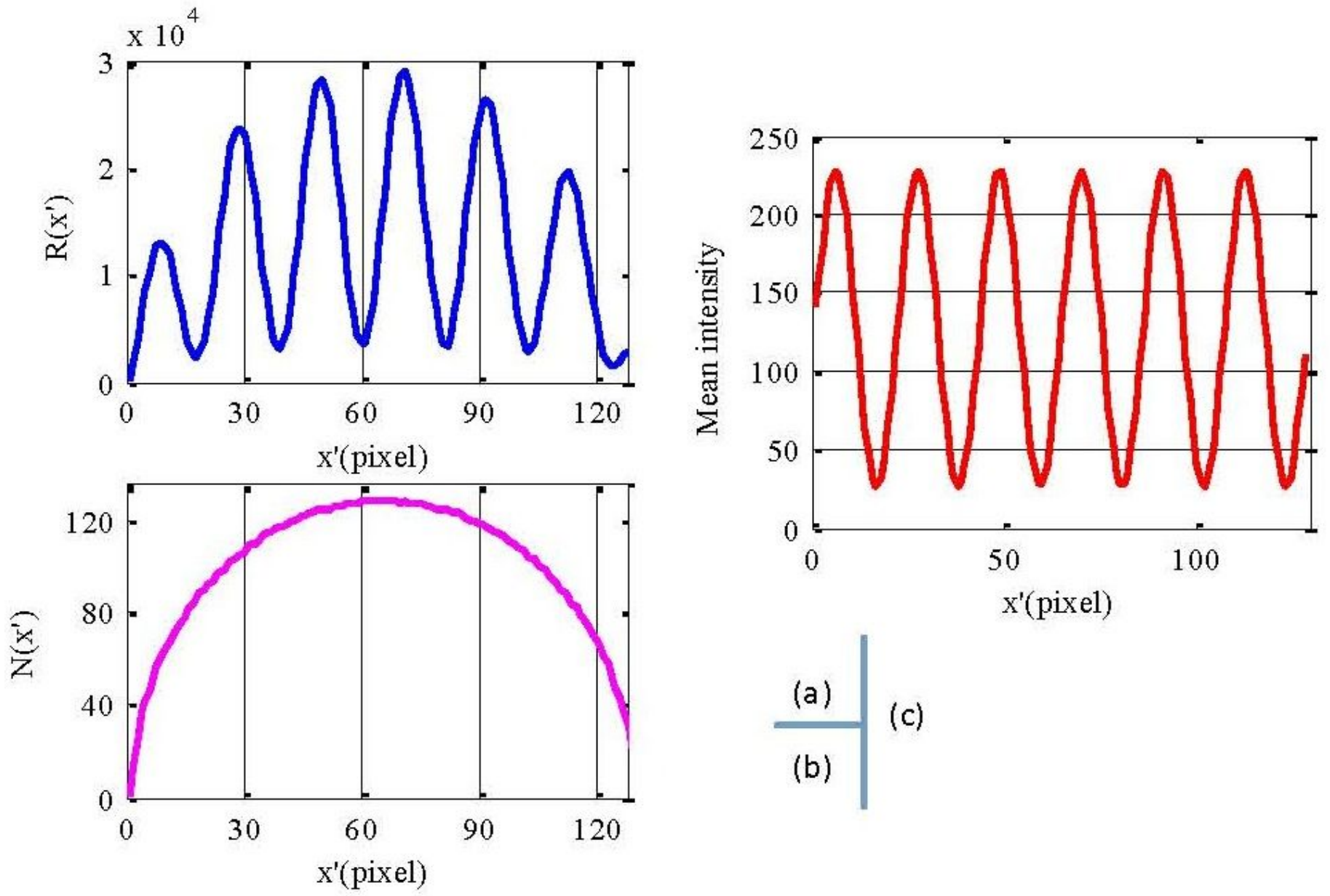

Figure 4

Accumulated intensities (a), pixel's number (b) and mean intensities (c) along the fringe direction.

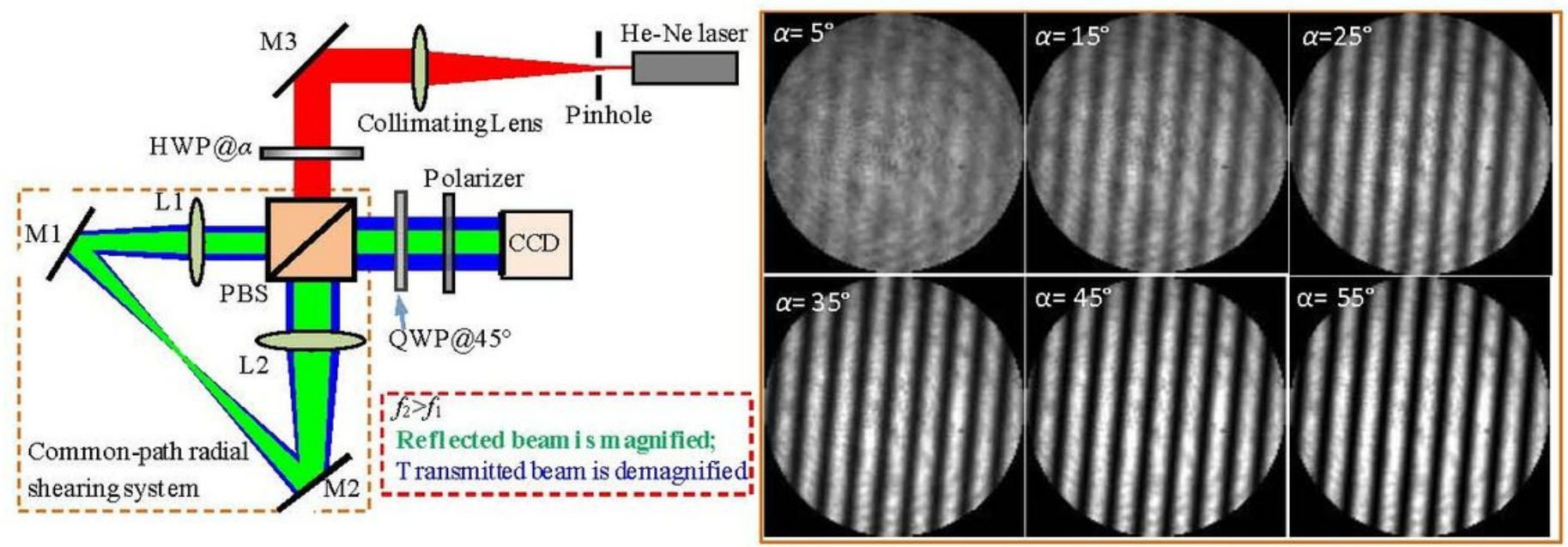

Figure 5

Experimental setup of common-path radial shearing interferometer and the experimental patterns under different rotating angle of HWP. HWP, half wave plate; PBS, polarizing beam splitter; L1-L2, lenses; M1-M3, 
mirrors; QWP, quarter-wave plates

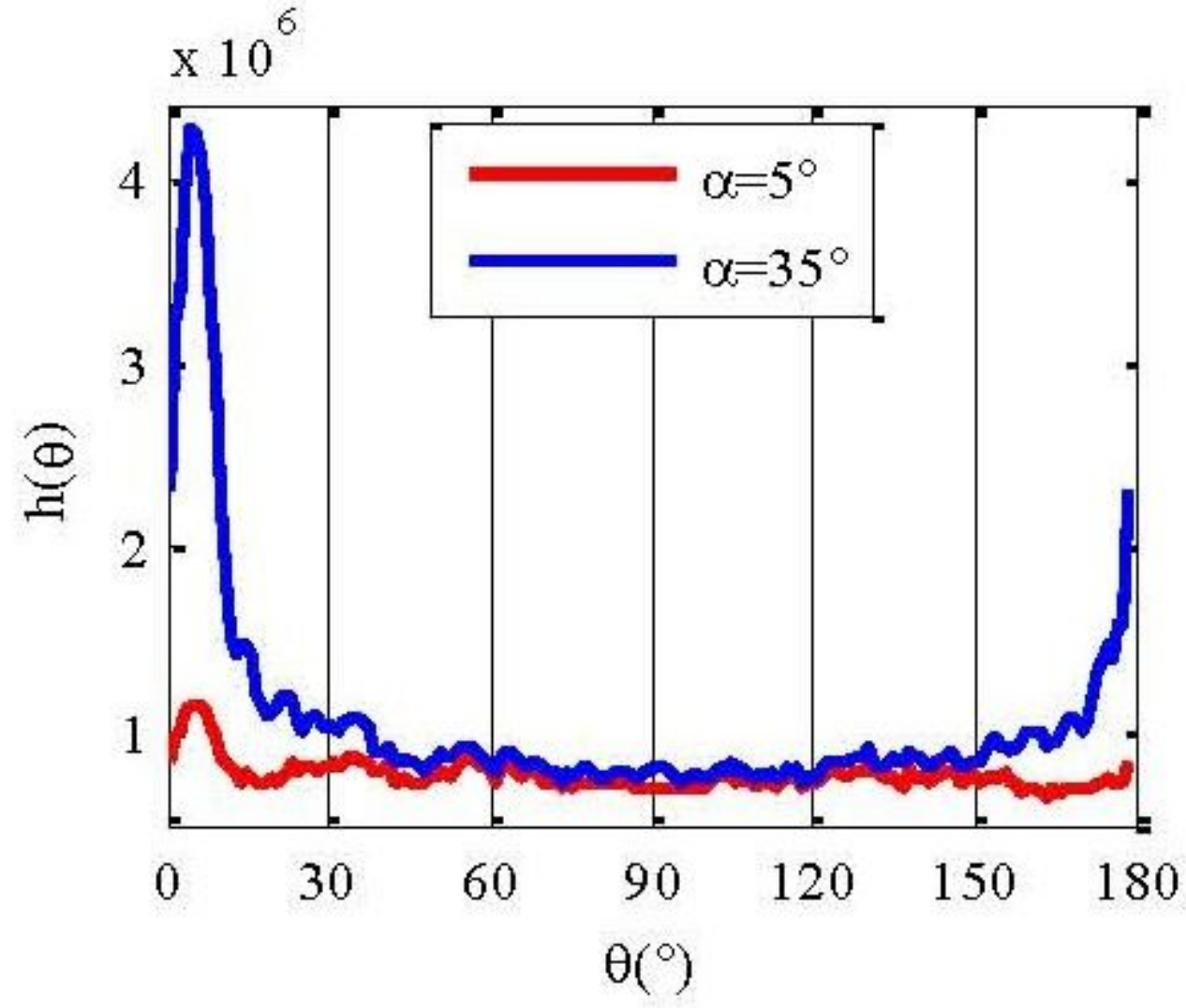

Figure 6

$h(\theta)$ curves of two patterns shown in Fig.5(right).

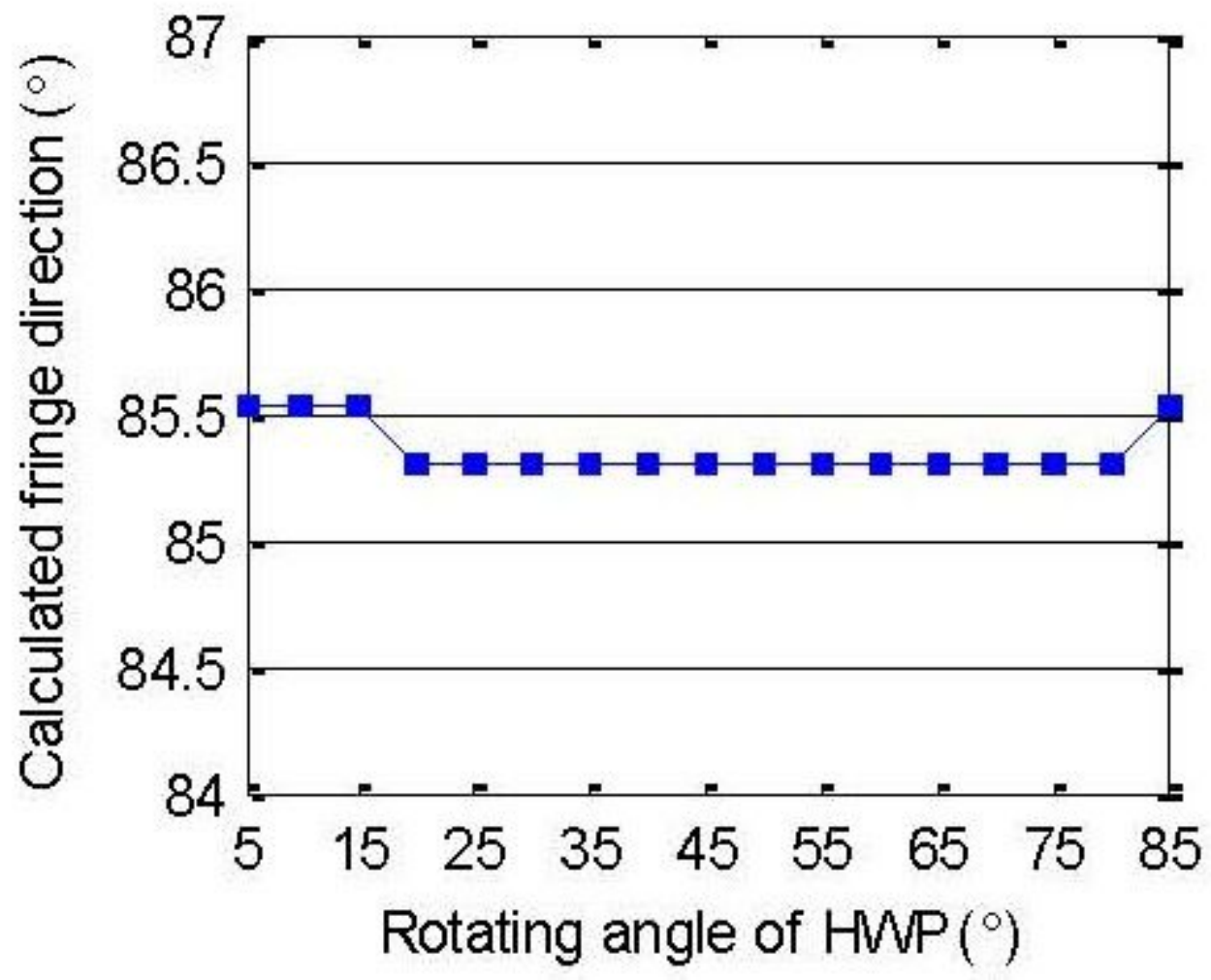


Figure 7

Fringe direct ions calculated for all experimental patterns.

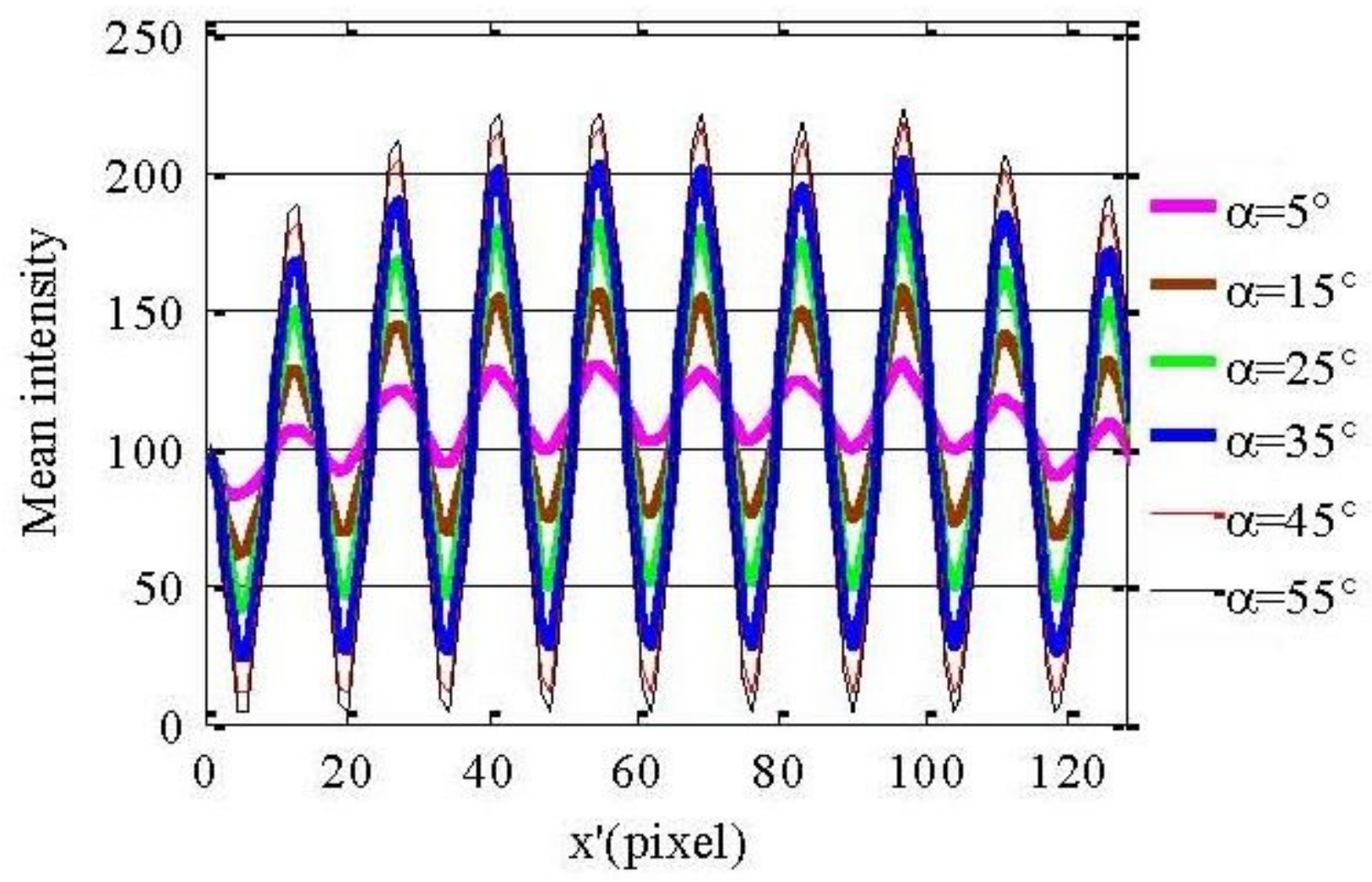

Figure 8

Mean projection intensities of six patterns shown in Fig.5(right) 


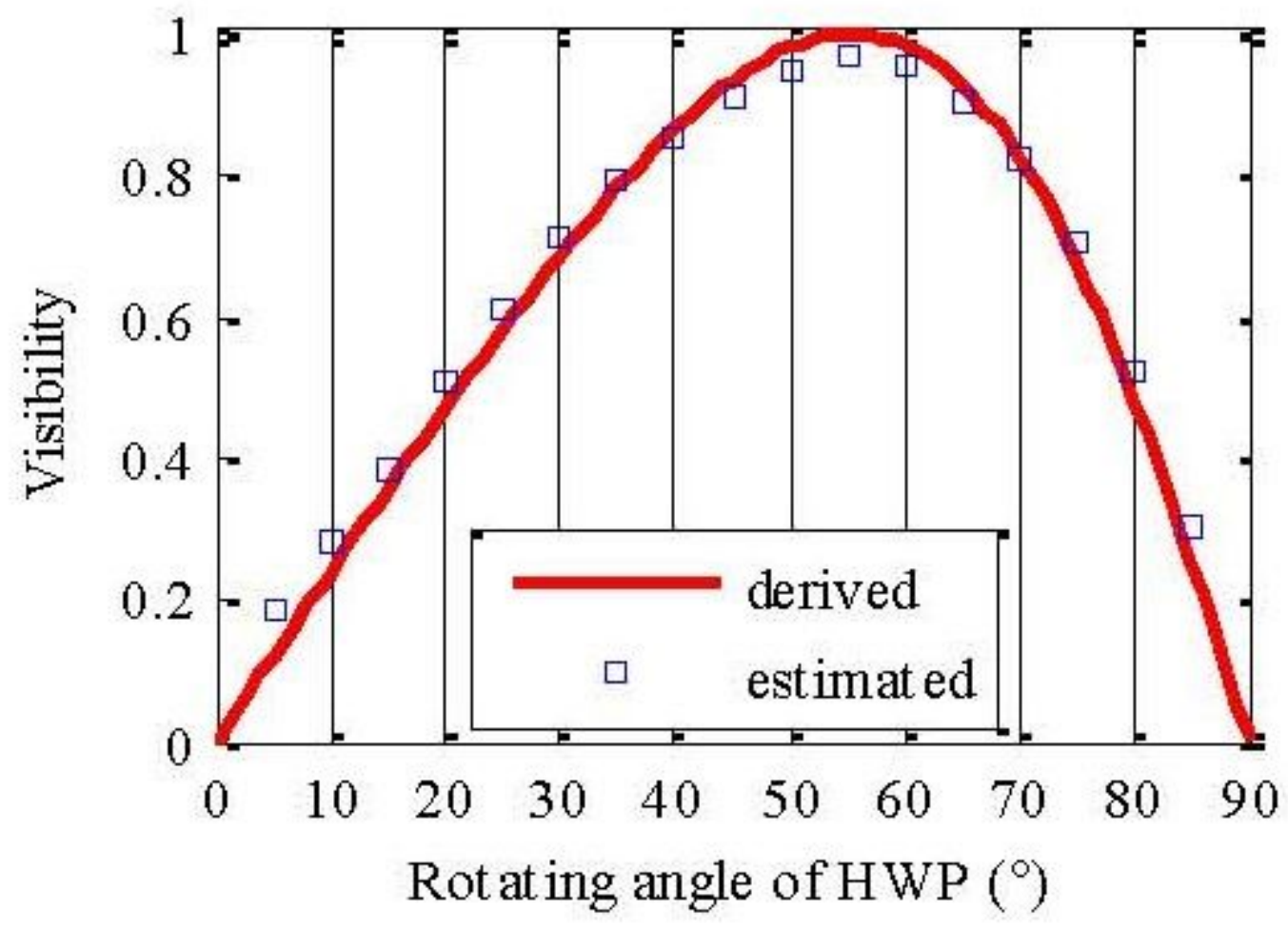

Figure 9

The est imated visibility from experimental patterns and the derived visibility from theoretical model. 